\title{
TWO CARRIERS FOR MOTION PERCEPTION: COLOR AND LUMINANCE
}

\author{
Thomas V. Papathomas, ${ }^{1, *}$ Andrei Gorea ${ }^{2}$ and Bela Julesz ${ }^{3}$ \\ 'Visual Perception Research Department, AT\&T Bell Laboratories, Murray Hill, NJ 07974, U.S.A., \\ ${ }^{2}$ Laboratory of Experimental Psychology, René Descartes University and CNRS, 75006 Paris, France and \\ ${ }^{3}$ Laboratory of Vision Research, Department of Psychology, Rutgers University, New Brunswick, \\ NJ 08903, U.S.A.
}

(Received 1 June 1990; in revised form 13 March 199I)

\begin{abstract}
Starting with the experiments of Ramachandran and Gregory (Nature, 275, 55-56, 1978), several psychophysical studies in apparent motion (AM) have established that the perception of motion is significantly impaired at equiluminance. Still debated, however, is whether color alone can resolve ambiguities in AM. We report here on several psychophysical experiments, the quantitative results of which indicate that color does play a substantial role in AM. These findings seem to support recently proposed neurophysiological frameworks according to which there exist significant interactions among the neuronal pathways mediating the perception of basic visual attributes such as color, motion, form and depth.

Color Luminance Apparent motion Equiluminance
\end{abstract}

\section{INTRODUCTION}

The main finding in the experiments of Ramachandran and Gregory (1978) was that apparent motion (AM) was severely impaired with random-dot cinematograms at equiluminance, the situation in which the two types of dots are discriminated from each other by variations in wavelength (color) but not in luminance. Since then, several studies have reported that the contribution of color to AM is weaker than that of luminance (Moreland, 1980; Kelly, 1983; Cavanagh, Tyler \& Favreau, 1984; Cavanagh, Boeglin \& Favreau, 1985; Derrington \& Badcock, 1985; Mullen, 1985; Mullen \& Baker, 1985; Cavanagh \& Anstis, 1986; Troscianko, 1987; Sato, 1988; Troscianko \& Fahle, 1988). There has been recent psychophysical evidence that color plays a role in AM (Gorea \& Papathomas, 1987, 1989; Sato, 1988; Green, 1989). Some researchers, however, have proposed that color cannot provide any input at all to motion perception mechanisms, and they argue that color and motion information are processed by separate, parallel pathways (Srinivasan, 1985; Livingstone \& Hubel, 1987, 1988; Carney, Shadlen \& Switkes, 1987). Although there is general agreement that color-

"Current address: Laboratory of Vision Research, Rutgers University, New Brunswick, NJ 08903, U.S.A. induced AM appears slower than luminanceinduced $\mathrm{AM}$ of equal objective speed (Cavanagh et al., 1984; Livingstone \& Hubel, 1987; Troscianko \& Fahle, 1988), there is still a debate as to whether color plays any role at all in motion perception. A central question in this debate, raised by Livingstone and Hubel (1987), is whether color alone can resolve ambiguities in AM. The experiments described in this paper were designed to investigate this question and the results indicate that the answer is affirmative.

\section{RATIONALE—STIMULI}

Stimuli of the type shown in Fig. 1 have been used by several visual psychophysicists to study the contribution of an attribute to motion (see, for example, Burt \& Sperling, 1981). The stimuli in Fig. 1 are shown in a schematic form in the $x-t$ space (Adelson \& Bergen, 1985). The spatial variable $x$ increases horizontally to the right and the temporal variable increases vertically downward. Although frames 1 and 2 are physically shown below frame 0 in Fig. 1, they are spatially superimposed over time in the actual experiments; frame $n$ is, of course, erased before frame $n+1$ is displayed. In each frame the elements (targets) occupy positions that are periodic in $x$ with period $P_{x}$. If all the elements 


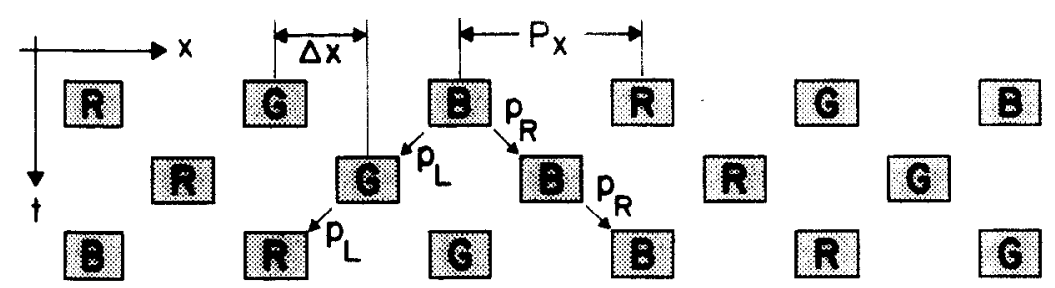

Fig. 1. Schematic representation of motion stimuli in the $x-t$ domain. The inter-frame displacement $\Delta x$ is $P_{x} / 2$. The symbols $\mathrm{R}, \mathrm{G}$ and $\mathrm{B}$ denote red, green and blue, respectively. The color is matched in the spatiotemporal domain to elicit movement perception to the right, while the luminance of all the elements is fixed. We term this condition $C w L$ (color within luminance).

are identical (the situation in Fig. 1 when one ignores the $R, G, B$ labels), the direction of motion is ambiguous since the inter-frame displacement, $\Delta x$, is half the spatial period $P_{x}$; this results in equally probable movement paths to the left $\left(p_{\mathrm{L}}\right)$ or to the right $\left(p_{\mathrm{R}}\right)$. To study the role of a single attribute in $\mathrm{AM}$, one may attempt to break the ambiguity in direction by matching that attribute in the spatiotemporal domain (Burt \& Sperling, 1981), as indicated by the $R, G, B$ symbols, to produce rightward motion. If such a stimulus elicits coherent motion perception, it follows that this particular attribute is a token for motion under the specific spatiotemporal conditions. We term the condition of Fig. 1 as matching of color within luminance, since color is spatiotemporally matched to elicit AM, while luminance is held constant.

In the special case where the attribute under study is color, one can make the background equiluminant to the elements in order to avoid unwanted motion signals due to variations in luminance. Equiluminance, however, is very difficult to achieve because it varies from observer to observer and it is also a function of retinal eccentricity (Livingstone \& Hubel, 1987). Thus, even if the stimulus of Fig. 1 with equiluminant background elicits motion perception, one may argue that this is due not to the color differences, but to the slight luminance residual differences among the red, green and blue elements. To circumvent the problems with equiluminance, we devised a set of stimuli, based on the class of multi-attribute stimuli of Papathomas and Gorea (1988), which allow us to study the role and interactions of color and luminance in AM.

\section{Stimuli}

The properties of the class of multi-attribute motion stimuli that we employed in this study are described in detail elsewhere (Papathomas \& Gorea, 1988). Basically, their main advantageous feature is that they permit each attribute (color, luminance, spatial frequency, orientation, etc.) to be matched in the $x-t$ plane simultaneously with, but independently of, the rest of the attributes. This, in turn, makes it possible to study the interaction of several attributes in motion perception and, in particular, it allows a direct comparison of the relative strength of two attributes, as explained below [see Fig. 2(d) and Experiment 3(a)].

The two attributes studied in this paper are color and luminance. The particular members of our class of stimuli are shown schematically in Fig. 2. The targets are defined by the conjunction of three different colors (red, R; green, $\mathrm{G}$; and blue, B) and three luminance values (low, $L_{\min }$, denoted by hatched areas in Fig. 2; medium, $L_{\text {med }}$, denoted by dotted areas; high, $L_{\max }$, denoted by white areas). The spatiotemporal distributions of the values for color and luminance for the purpose of eliciting motion perception are independent from each other and they give rise to the following important special types of stimuli.

Color across luminance. This arrangement, which we denote by $C \times L$, is shown in Fig. 2(a). Here, color is matched as in Fig. 1, to produce coherent motion to the right, but luminance varies cyclically among three widely different values along both, the leftward and the rightward paths, without contributing to coherent motion. This stimulus was used in Experiment 1 to test whether color is a token for motion perception.

Luminance across color $(L \times C)$. This is the dual of the previous arrangement $(C \times L)$, in which the roles of color and luminance are interchanged. This is shown schematically in Fig. 2(b), in which luminance is matched to produce $\mathrm{AM}$ to the right, while color is arranged cyclically along both, the leftward 


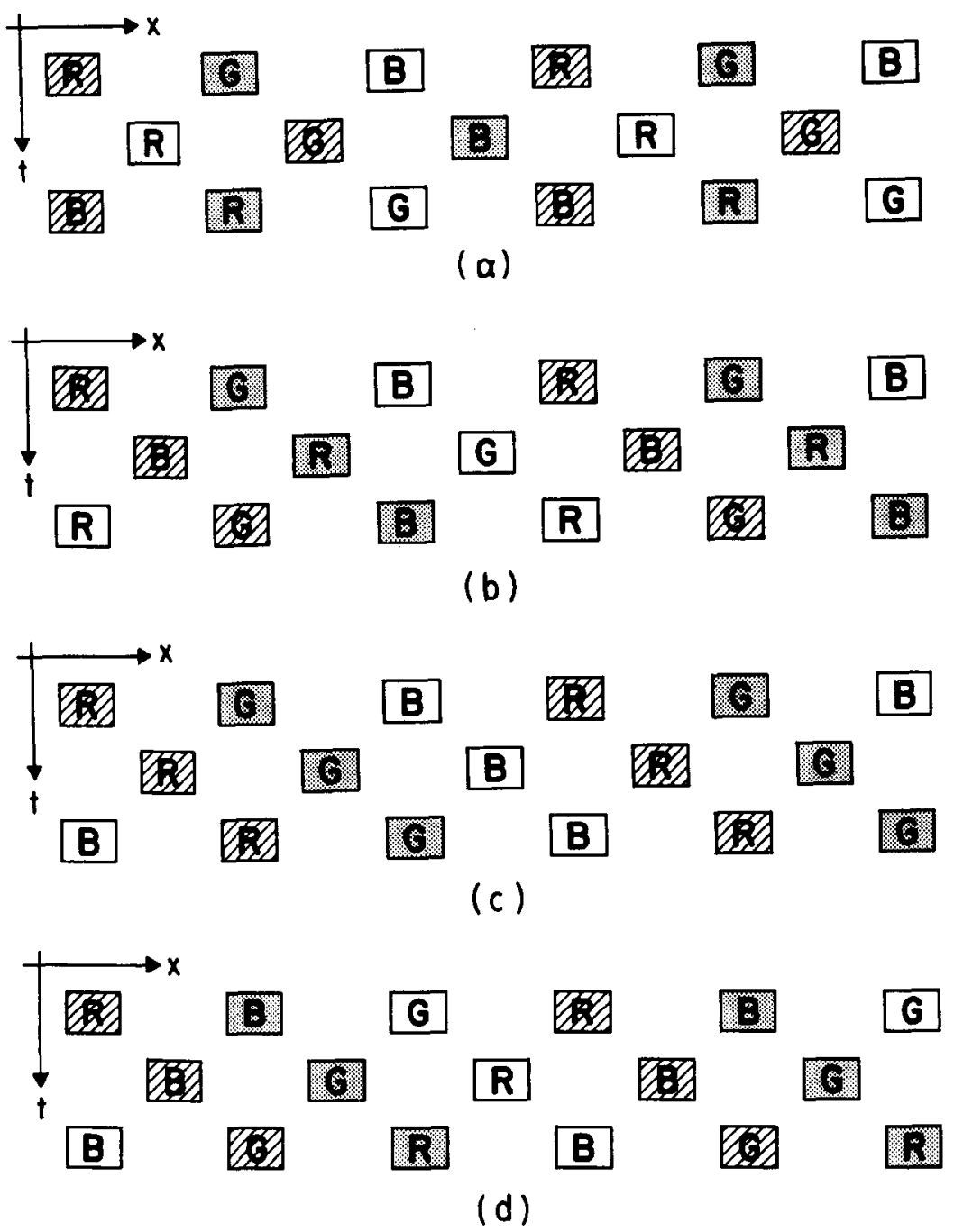

Fig. 2. Schematic representation of the stimuli used in the present study. The same conventions apply as in Fig. 1. White, dotted and hatched areas denote elements of high, medium and low luminance, respectively. (a) Color is matched as in Fig. 1, but luminance varies cyclically along both the left- and right-ward paths, i.e. color across luminance $(C \times L)$. (b) Luminance is matched to produce rightward motion, while color varies cyclically $(L \times C)$. (c) Both color and luminance are matched to elicit movement perception to the right $(C+L)$. (d) Color and luminance are matched, to produce motion in opposing directions, i.e. color against luminance $(C \leftrightarrow L)$.

and the rightward paths, thus contributing no coherent motion signal.

Color plus luminance $(C+L)$. In this arrangement, shown in Fig. 2(c), both attributes are matched in the spatiotemporal domain to elicit unambiguous motion to the right.

Color against luminance $(C \leftrightarrow L)$. In this scheme, shown in Fig. 2(d), color is arranged in the $x-t$ plane to elicit coherent motion to the left while, simultaneously and independently, luminance is arranged to produce motion to the right. This is an example of a stimulus which allows the direct comparison of the relative strength of two attributes in motion perception.

\section{METHODS}

\section{Stimuli}

The stimuli were generated on a Digital Equipment Corporation VAX11/750 computer. They were stored and displayed by an ADAGE RDS 3000 raster frame buffer. Images were displayed on a Sony Trinitron color monitor (PVM-1271Q) on a dark background, $120 \mathrm{~cm}$ from the observer. At that distance, the width and height of the rectangular elements (targets) subtended $0.38^{\circ}$ and $0.29^{\circ}$ of visual angle, respectively. The CIE $x, y$ coordinates for red, green and blue were $(0.65,0.31),(0.29,0.59)$ and $(0.14,0.05)$, respectively. These were measured 
with a Minolta Color Analyzer II, model TV/2130. The average values of the three luminance levels were $3.0,8.4$ and $23.2 \mathrm{~cd} / \mathrm{m}^{2}$. The background was dark (below $0.01 \mathrm{~cd} / \mathrm{m}^{2}$ ) in all the experiments. The interframe displacement $\Delta x$ was $0.5^{\circ}, 0.72^{\circ}, 1.0^{\circ}$ and $1.15^{\circ}$, depending on the experimental condition. The inter-element distance within a frame, measured from center to center of adjacent elements, was always twice the value of $\Delta x$. The frame duration was $33.33 \mathrm{msec}$ with no dark interstimulus interval (ISI). Four element-rows, rather than one [as shown schematically on Fig. 2(a)], were displayed simultaneously in each frame. For a given stimulus type (say, $C+L$ ), each of these four rows is subjected to the corresponding transformation indicated in Fig. 2 [Fig. 2(c) for $C+L]$. Each row's horizontal position was randomly jittered to prevent any spatial structuring (Gorea \& Papathomas, 1987; Papathomas \& Gorea, 1988); basically, instead of placing the leftmost element of a row at $x=0$, we added a random displacement, uniformly distributed between 0 and $P_{x}$. A typical four-row frame is shown in Fig. 3 for the special case of $\Delta x=0.72^{\circ}$; the conventions for color and luminance are the same as those used in Figs 1 and 2. The vertical space from the bottom of one row to the top of the row below it is $0.21^{\circ}$. One image-frame subtended $9.1^{\circ}$ horizontally and $2^{\circ}$ vertically. A fixation cross was placed at the center of the display to minimize eye movements. It consisted of two white lines each approx. $0.17^{\circ}$ long and $1.15^{\prime}$ wide, crossing perpendicularly at their midpoint; its luminance was $32.3 \mathrm{~cd} / \mathrm{m}^{2}$.

\section{Procedure}

Equiluminant settings. To account for interobserver differences, we controlled the luminance levels in all experiments in order to match each observer's individual characteristics. The luminance of the red, green and blue elements was matched for each of the three observers that took part in the experiment at the low luminance setting, so that the three "dim" elements of different color were equiluminant among themselves. This matching was repeated for three medium-luminance and for the three high-luminance elements. Equiluminance was obtained using the flicker photometry method. Here is a brief outline of the procedure, which is described in detail elsewhere (Gorea \& Papathomas, 1989). A magenta background with CIE $(x, y)$ coordinates $(0.25,0.14)$ was used as background; its luminance was set at the desired reference level $\left(L_{\min }, L_{\operatorname{med}}\right.$ or $\left.L_{\max }\right)$. An array of elements, identical in size with the targets used in the experiment, was displayed on the background. All the elements were of the same test color (R, G or B); the objective was to obtain an equiluminant setting for the test color with respect to the background. The colors of the background and the elements were alternated for six times at a rate of $30 \mathrm{~Hz}$. After each series of six alternations, the observer adjusted the luminance of the elements until he/she arrived at a setting which minimized the perceived flicker. This procedure was repeated at least five times for each observer and for each target color and the values were averaged to obtain each observer's equiluminant setting for $\mathrm{R}, \mathrm{G}$ and $\mathrm{B}$. The SD for the five (or more) equiluminant settings for a given observer and a fixed color and luminance level was not greater than $4.8 \%$, with an average value of $1.85 \%$. Variations among observers were greater: the mean equiluminant settings varied by less than $15.0 \%$ among observers and had a SD of at most $8.8 \%$ (the average value was $4.03 \%)$.

Main experiments. One of the authors (TVP) and two naive observers (DD and CK) were the observers in all experiments. The direction of motion was randomly changed from trial to trial. The observer's tasks was to report on the

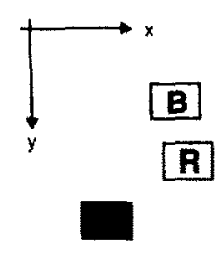

R
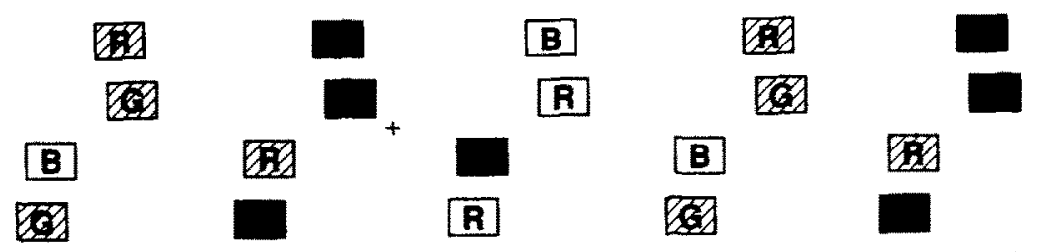

Fig. 3. An example of a single image-frame used in the experiments. Four rows were displayed simultaneously. Elements are spaced uniformly along the vertical and horizontal directions. The horizontal position of the leftmost element of each row was rondomized to prevent the formation of regular patterns. 
direction (leftward or rightward) in a twoalternative, forced-choice (2AFC) paradigm. The length of the animation sequence ranged from 2 to 4 frames ( 3 conditions) and it was randomized across trials. In general, direction discrimination performance improved as the sequence length increased. Since the relative performance as a function of stimulus type $(C \times L, L \times C, C+L$, and $C \leftrightarrow L)$ was not affected by the sequence length, performances are presented by averaging the results of the three sequence values. One session consisted of 150 trials, 50 per sequence length. The value of $\Delta x$ was held fixed within a session and varied randomly across sessions. There were at least 3 sessions per observer for each value of $\Delta x$, resulting in at least 150 trials per condition per observer. Since the duration of each frame was $33.33 \mathrm{msec}$, the longest sequence was $133.33 \mathrm{msec}$, thus not allowing initiation of eye movements. Discrimination of the direction of motion was recorded for each combination of observer, $\Delta x$, and length of sequence. Unless otherwise indicated, the parameter values used in all the experiments were as described above.

The rationale and types of stimuli for individual experiments are briefly outlined below.

\section{Experiment 1}

The purpose of this experiment was to investigate whether color can resolve ambiguities in AM. Accordingly, the stimulus type $C \times L$ [Fig. 2(a)] was used, in which color is arranged spatiotemporally to elicit motion perception across luminance. Luminance varies widely, but its arrangement is meant to produce an ambiguous direction of motion, as shown in Fig. 2(a).

\section{Experiment 2}

This consisted of a pair of experiments. The first one was conducted with the stimulus type $L \times C$, shown schematically in Fig. 2(b); the second one employed the stimulus type $C+L$ [Fig. 2(c)]. For both stimuli, luminance is matched to produce unambiguous AM. The only difference is that color is arranged cyclically in Fig. 2(b) [Experiment 2(a)], thus contributing no additional motion signal, whereas in Fig. 2(c) [Experiment 2(b)], color is also matched to elicit motion to the right, thus attempting to enhance the contribution of luminance. Thus, the gain in performance, if any, for $C+L$ over $L \times C$ must be attributed mainly to the contribution of color. For an alternative way of interpreting this gain in performance, see the Discussion section.

\section{Experiment 3}

Three different experiments were conducted under this category. In Experiment 3(a) we used the stimulus type $C \leftrightarrow L$ [Fig. 2(d)], in which color and luminance compete against each other, because they are arranged spatiotemporally to elicit AM in opposite directions. We attempted to find conditions for which color would dominate over luminance by gradually weakening the strength of the latter. It is obvious that, as the luminance ratio $L_{\max } / L_{\min }$ is decreased in Fig. 2(d) (we kept the value of $L_{\text {med }}$ constant), the strength of luminance-elicted motion also diminishes, as compared to that of color. In Experiment 3(a) when we started with a high value for $L_{\max } / L_{\min }$, all observers reported the direction of motion to be dominated by luminance matching with the stimulus of Fig. 2(d). However, as we decreased $L_{\max } / L_{\min }$, there came a point, which we call $L_{\max }^{*} / L_{\min }^{*}$, for which the direction was clearly dominated by color matching. This point varied from observer to observer. The critical question is: was the color dominance over a significant luminance contribution, or were $L_{\min }^{*}$ and $L_{\max }^{*}$ such as to render the luminance strength virtually nonexistent? To answer this question, we conducted Experiment 3(b) with the stimuli of Fig. 2(b), i.e. luminance across color $(L \times C)$ using $L_{\min }^{*}$ and $L_{\max }^{*}$. The performance of observers in this condition gives us the strength of luminance without matched color and helps us answer the above question. Finally, for completeness, we also conducted Experiment 3(c) with the stimuli of Fig. 2(a) $(C \times L)$ to find the strength of chromatic -matching across luminance, using $L_{\min }^{*}$ and $L_{\max }^{*}$. Since $L_{\min }^{*}$ and $L_{\max }^{*}$ varied from observer to observer, these were the only parameter values that were different from those used in Experiments 1 and 2.

\section{RESULTS}

\section{Experiment I}

The results from experiments with the stimulus of Fig. 2(a) $(C \times L)$ are shown in Fig. 4 by solid circles. The abscissa is $\Delta x$ and the ordinate is the success rate (percent correct) of judging the direction of motion. There were no significant statistical variations across observers with respect to the relative strength of motion resulting from the stimuli of Fig. 2(a), (b) and (c) [the 


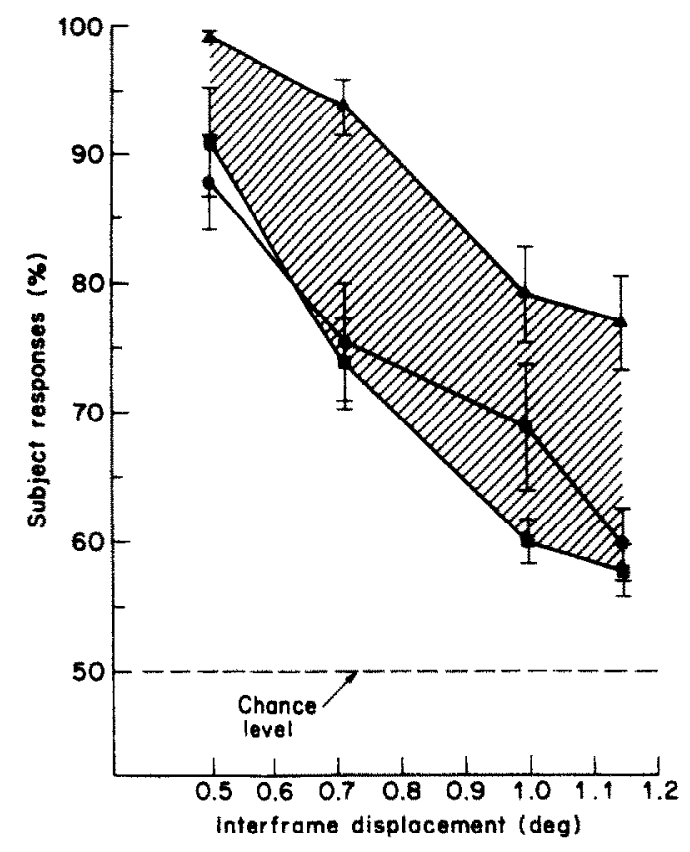

Fig. 4. Percentages of correct discrimination of motion direction, averaged across the three observers, as a function of inter-frame displacement. Vertical bars show standard errors ( $\pm 1 \mathrm{SE}$ ). Change level is at $50 \%$. Circles denote results with stimuli similar to that of Fig. 2(a) in which motion is "carried" by color, while luminance is not matched to produce coherent movement. The results in the dual condition, i.e. motion produced by luminance while color is not matched [Fig. 2(b)] are shown by square symbols. Triangles indicate results obtained when both luminance and color are matched to produce movement in the same direction [Fig. 2(c)].

results with Fig. 2(b) and (c) are explained in Experiment 2 below]. This is why each point was obtained by averaging the results of the three observers for the three different sequence lengths and it represents the average of at least 1350 trials. It is clear from the graph shown in solid circles in Fig. 4 that color can indeed resolve ambiguities in AM perception.

This result was also corroborated in a separate experiment, in which color was matched to produce coherent motion, as in Fig. 2(a), but the luminance of each element was assigned randomly among 60 discrete values, in a uniform distribution ranging from 6.0 to
$19.2 \mathrm{~cd} / \mathrm{m}^{2}$. The percent correct responses, averaged for observers TVP and DD, were 96.8 , 81.0 and 66.8 for displacement values $(\Delta x)$ of $0.5^{\circ}, 0.72^{\circ}$ and $1.0^{\circ}$, respectively, for this experiment.

The presence of luminance- and color-defined edges must be noted in our stimuli. Thus, our stimuli reveal the ability of color matching to resolve ambiguities in the presence of luminance-defined edges. The fact that such edges are not present in random-dot cinematogram (RDC) stimuli, used by Chang and Julesz (1989) may account for the differences between our findings and theirs. On the other hand, as previously shown by Cavanagh et al. (1984), color-induced motion impairment is minimal for stimuli that contain high spatial frequencies or move at high speeds. Moreover, we also conducted experiments with equiluminant background and elements of a uniform luminance (see Fig. 1), in which motion discrimination performances were significantly above the chance level (see also Gorea \& Papathomas, 1989).

\section{Experiment 2}

Additional evidence for the role of color in AM is provided by the pair of Experiments 2(a) and (b). The results of Experiment 2(a) $(L \times C)$ are shown in Fig. 4 as squares and those of Experiment 2(b) $(C+L)$ are indicated by triangles. Performances in the $L \times C$ condition (squares) are comparable to those in the $C \times L$ condition (circles). Notice the relatively high luminance range $\left(3.0-23.2 \mathrm{~cd} / \mathrm{m}^{2}\right)$ needed to obtain this parity in performance. When we compare the $C+L$ to the $L \times C$ performance, it is clear that the added signal due to color contributes significantly to the strength of the AM due to luminance alone. The shaded area is meant to show the gain in performance due to color. The data from the two experiments with Fig. 2(b) and (c) were subjected to an analysis-of-variance (ANOVA) test which confirmed that the effect of color in

Table 1. The results of the analysis-of-variance test on the data of Experiment 2

\begin{tabular}{lccccc}
\hline $\begin{array}{l}\text { Sources of } \\
\text { variability }\end{array}$ & $\begin{array}{c}\text { Degrees of } \\
\text { freedom }\end{array}$ & $\begin{array}{c}\text { Sums of } \\
\text { squares }\end{array}$ & $\begin{array}{c}\text { Mean } \\
\text { squares }\end{array}$ & $\begin{array}{c}F \\
\text { factors }\end{array}$ & $\begin{array}{c}\text { Significance } \\
\text { levels }\end{array}$ \\
\hline $\begin{array}{l}\text { Observers } \\
\text { Inter-frame }\end{array}$ & 2 & 0.1309 & 0.06545 & 3.475 & 0.067 \\
$\quad \begin{array}{l}\text { displacements } \\
\text { Presence of color }\end{array}$ & 3 & 2.4494 & 0.8165 & 43.36 & $<0.001$ \\
Residuals & 1 & 1.1582 & 1.1582 & 61.50 & $<0.001$ \\
Total & 65 & 1.2241 & 0.01883 & & \\
\hline
\end{tabular}



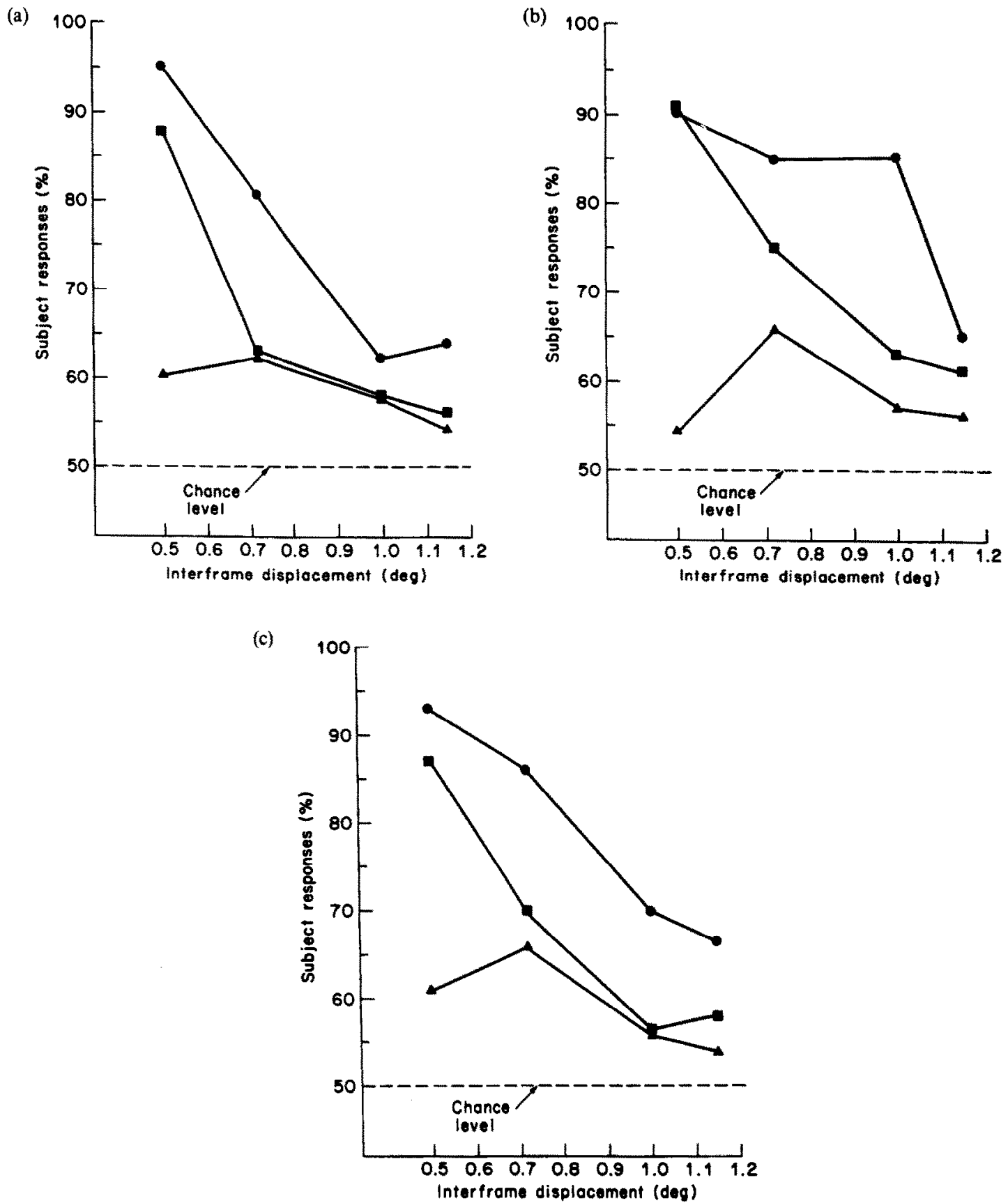

Fig. S. In each graph, the circles denote percentages of correct discrimination for the direction of motion due to color [stimulus of Fig. 2(a)] as a function of inter-frame displacement. Squares indicate the same performance index for motion due to luminance [stimulus of Fig. 2(b)]. Triangles indicate percentages of responses in which the perceived direction was dominated by color-elicited motion when stimuli similar to those of Fig. 2(d) were employed. (a) Observer TVP: $L_{\min }^{*}=5.1, L_{\bmod }=8.4, L_{\max }^{*}=14.5 \mathrm{~cd} / \mathrm{m}^{2}$. (b) Observer DD: $L_{\min }^{*}=3.0, L_{\text {med }}=8.4, L_{\max }^{*}=23.2 \mathrm{~cd} / \mathrm{m}^{2}$. (c) Observer $\mathrm{CK}: L_{\min }^{*}=4.9, L_{\operatorname{med}}=8.4$, $L_{\max }^{*}=16.2 \mathrm{~cd} / \mathrm{m}^{2}$.

AM is highly significant $[P \ll 0.001$; percent correct $(\%)$ transformed to arcsin $(\sqrt{\% \text { correct }})$ prior to the analysis of variance]. There is an improvement of 17.56 percentage points (SE 2.05 ). The results of the statisitical analysis are summarized in Table 1.

\section{Experiment 3}

The results from Experiment 3(a) $(C \leftrightarrow L)$, Experiment 3(b) $(L \times C)$ and Experiment 3(c) $(C \times L)$ are shown in Fig. 5 by triangles, squares and circles, respectively. Since the 
values of $L_{\min }^{*}$ and $L_{\max }^{*}$ (for which the chromatic contribution to AM dominates that of luminance) are different for the three observers, the results are shown in separate graphs for each observer [Fig. 5(a), (b), and (c) for TVP, DD and CK, respectively].

Each point in Fig. 5 was obtained by averaging the results of the observer for the three different sequence lengths and it represents the average of at least 450 trials. For the stimulus of Fig. 2(d) the triangles indicate the percentage of responses in which color dominated in determining the direction of motion. Notice that, for each observer, the luminance-elicited AM with cyclically varying colors [stimulus of Fig. 2(b)] produces strong discrimination of direction [square symbols in Fig. 5(a), (b) and (c)], especially for small $\Delta x$. When, however, color was matched to produce AM in the opposite direction [stimulus of Fig. 2(d)], the perceived direction was dominated by the color component (triangular symbols) for the particular values of $L_{\min }^{*}, L_{\operatorname{med}}$ and $L_{\max }^{*}$ indicated for each observer in the legend of Fig. 5. $L_{\text {med }}$ was fixed for all experiments at $8.4 \mathrm{~cd} / \mathrm{m}^{2}$. Notice that performance for stimulus type $C \times L$ is better than that for $L \times C$; hence one would expect color to dominate over luminance in the $C \leftrightarrow L$ experiment. What we demonstrated here, however, is that there is a luminance range (varying from observer to observer) for which the direction of motion is dominated by the chromatic component at the expense of the luminance component (triangles in Fig. 5), even though the same luminance range produces direction discrimination performances that are significantly above chance level (squares in Fig. 5) for the stimuli of Fig. 2(b).

\section{DISCUSSION}

First we offer a few comments on the issue of equiluminance. Even if the red, green and blue targets are not truly equiluminant due either to imperfect matching of the flicker photometry procedure or to retinal eccentricity, we do not think this to be critical with respect to our conclusions. This is because the small deviations around a given level are negligible compared to the large differences between levels, such as $L_{\max }-L_{\text {med }}$ and $L_{\text {med }}-L_{\min }$. Thus, our stimuli are less sensitive to deviations around the equiluminant setting.

There is a potential interaction between the effects of $\Delta x$ on performance and simply the number of elements per row as $\Delta x$ is varied, since the overall field size is held constant across variations in $\Delta x$. Given our stimulus dimensions, the average number of tokens per row was 9 and 3.5 for the minimum and maximum $\Delta x$, respectively. This corresponds roughly to 2.7 and 0.88 visible cycles, where one cycle corresponds to either a Red-Green-Blue or a dim-medium-high luminance spatial succession. Directional sensitivity has been indeed shown to depend on the number of visible cycles in this range (Gorea, 1986) but this dependency is quite weak to account for the dramatic performance drop we report here.

Another way to interpret the gain in performance with the $C+L$ type of stimuli over that of the $L \times C$ type, suggested by a reviewer, is to view the spatiotemporally correlated and uncorrelated attributes as signal and noise, respectively. The correlated attribute is arranged so as to elicit unambiguous, coherent motion; the uncorrelated one is arranged cyclically in the $x-t$ plane. Then, the gain in performance can be viewed as reflecting absence of noise in the $C+L$ stimulus, rather than augmentation. This is a very reasonable interpretation. However, experiments in progress in our laboratories where one attribute is correlated but the other is held constant (we refer to them as "within" conditions; e.g. Fig. 1, color within luminance) result in performances that are equal or even worse than those obtained under "across" conditions. This is why we favor the interpretation that it is the correlation of color in the $C+L$ stimulus, in addition to the correlation of luminance, that results in the performance improvement over the $L \times C$ stimulus.

Taken together, our results indicate that color cues can be added to break the ambiguity of incoherent motion patterns (Experiment 1), or enhance the perception of coherently moving targets (Experiment 2) and even to dominate luminance-induced motion (Experiment 3). These data quantify and extent the finding of Ramachandran and Gregory (1978) that color is indeed a token for movement in experiments with line targets. They reported that AM perception disappeared at equiluminance with RDC. If, however, successive frames of equiluminant $\mathrm{RDCs}$ are not separated by dark inter-frame intervals, AM perception is still observed (Cavanagh et al., 1985). This observation, together with similar ones, contained in a comprehensive study of a wide range of AM phenomena, led Cavanagh and Mather 
(1990) to argue in favor of a single AM process, rather than the two separate (short-range and long-range) AM processes proposed earlier (Braddick, 1974; Pantle \& Picciano, 1976). Even if this dichotomy is valid, our stimuli are indeed difficult to classify as either shortor long-range for the following reasons: (1) The size of $\Delta x$ in our stimuli might classify them as long-range, although the relatively large size of the elements tends to blur this classification, since the maximum size of $\Delta x$ for which $A M$ can be perceived scales linearly with the size of the elements (Cavanagh $e t$ al. 1985). (2) We observed that the higher the spatial density of the elements, the better the performance. This would suggest the contribution of short- rather than long-range mechanisms. (3) The high rate of $30 \mathrm{~Hz}$ (and, most recently, $60 \mathrm{~Hz}$ in similar experiments) again suggests the activation of short-range mechanisms.

Psychophysical evidence linking chromatic input to motion mechanisms was presented, among others, by Cavanagh et al. $(1984,1985)$, Gorea and Papathomas (1987, 1989), Cavanagh (1987), Troscianko and Fahle (1988), Sato (1988), and Green (1989). Others present psychophysical evidence which suggests that color and motion pathways are separate (e.g. Livingstone \& Hubel, 1987; Carney et al., 1987). Neurophysiologists are also divided on this issue: Hubel and Livingstone (1987), for example, support the idea of separate pathways for color and motion, whereas DeYoe and Van Essen (1988), among others, argue that there are interactions between the two pathways. Recent neurophysiological experiments by Saito, Tanaka, Isono, Yasuda and Mikami (1989) and Logothetis, Schiller, Charles and Hurlbert (1990) indicate that there are mechanisms that respond selectively to moving equiluminous color stimuli. Our present psychophysical results also strongly suggest that there must be some interaction between neural mechanisms that subserve color and motion perception.

Acknowledgements-We thank P. Cavanagh for valuable suggestions. We also thank F. A. DeYoe and B. J. A. Kröse for stimulating discussions which resulted in this work, and D. Pregibon for expert statistical analyses on our data.

\section{REFERENCES}

Adelson, E. H. \& Bergen, J. R. (1985). Spatiotemporal energy models for the perception of motion. Journal of the Optical Society of America, 2A, 284-299.
Braddick, O. (1974). A short-range process in apparent motion. Vision Research, 14, 519-529.

Burt, P. \& Sperling, O. (1981). Time, distance and feature trade-offs in visual apparent motion. Psychological Review, 8, 171-195.

Carney, T., Shadlen, M. \& Switkes, E. (1987). Parallel processing of motion and color information. Nature, 328, $647-649$.

Cavanagh, P. (1987). Evaluating color vision with moving stimuli. Technical Digest Optical Society of America, 22, 51.

Cavanagh, P. \& Anstis, S. M. (1986). Do opponent-color channels contribute to motion? Investigative Ophthalmology and Visual Science (Suppl.), 27, 291.

Cavanagh, P. Mather, G. (1990). Motion: The long and short of it. Spatial Vision, 4, 103-129.

Cavanagh, P., Boeglin, J. \& Favreau, O. E. (1985). Perception of motion in equiluminous kinematograms. Perception, $14,151-162$.

Cavanagh, P., Tyler, C. W. \& Favreau, O. E. (1984). Perceived velocity of moving chromatic gratings. Journal of the Optical Society of America, A1, 893-899.

Chang, J. J, \& Julesz, B. (1989). A new method for testing color motion under randomized equiluminant condition. Investigative Ophthalmology and Visual Science, 30, 130.

Derrington, A. M. \& Badcock, D. R. (1985). The low level motion system has both chromatic and luminance inputs. Vision Research, 25, 1879-1884.

DeYoe, E. A. \& VanEssen, D. C. (1988). Concurrent processing streams in monkey visual cortex. Trends in Neurosciences, 11, 219-226.

Gorea, A. (1986) Spatial integration characteristics in motion detection and direction identification. Spatial Vision, 1, 85-102.

Gorea, A. \& Papathomas, T. V. (1987). Form and surface attributes in motion perception studied with a new class of stimuli: A basic asymmetry. AT \&T Bell Laboratories Technical Memorandum, 11223-870921-2TM. N.J.: Murray Hill.

Gorea, A. Papathomas, T. V. (1989). Motion processing by chromatic and achromatic pathways. Journal of the Optical Society of America, A6, 590-602.

Green, M. (1989). Color correspondence in apparent motion. Perception and Psychophysics, 45, 15-20.

Hubel, D. H. \& Livingstone, M. S. (1987). Segregation of form, color and stereopsis in primate area 18. Journal of Neuroscience, 7, 3378-3415.

Kelly, D. H. (1983). Spatiotemporal variation of chromatic and achromatic contrast thresholds. Journal of the Optical Society of America, 73, 742-750.

Livingstone, M. S. \& Hubel, D. H. (1987). Psychophysical evidence for separate channels for the perception of form, color, movement, and depth. Journal of Neuroscience, 7, 3416-3468.

Livingstone, M. \& Hubel, D. (1988). Segregation of form, color, movement and depth: Anatomy, physiology and perception. Science, 240,740-749.

Logothetis, N. K., Schiller, P. H., Charles, E. R. \& Hurlbert, A. C. (1990). Perceptual deficits and the activity of the color-opponent and broad-band pathways at isoluminance. Science, 247, 214-217.

Moreland, J. D. (1980). Spectral sensitivity measured by motion photometry. In Verriest, $G$. (Ed.), Color deficiencies VI (pp. 61-66). The Hague: Junk.

Mullen, K. T. (1985). The contrast sensitivity of 
human colour vision to red-green and blue-yellow chromatic gratings. Journal of Physiology, London, 359, 381-400.

Mullen, K. T. \& Baker, C. L. (1985). A motion aftereffect from an isoluminant stimulus. Vision Research, 25, 685-688.

Pantle, A. J. \& Picciano, L. (1976). A multi-stable movement display: Evidence for two separate motion systems in humans. Science, 193, 500-502.

Papathomas, T. V. \& Gorea, A. (1988). Simultaneous motion perception along multiple attributes: $A$ new class of stimuli. Behavioral Research Methods, Instruments and Computers, 20, 528-536.

Ramachandran, V. S. \& Gregory, R. L. (1978). Does color provide an input to human motion perception? Nature, 275, 55-56.

Saito, H., Tanaka, K., Isono, H., Yasuda, M. \& Mikami,
A. (1989). Directionally selective response of cells in the middle temporal area (MT) of the macaque monkey to the movement of equiluminous opponent color stimuli. Experimental Brain Research, 15, 1-14.

Sato, T. (1988). Direction discrimination and pattern segregation with isoluminant chromatic random-dot cinematograms (RDC). Investigative Ophthalmology and Visual Science, 29, 449.

Srinivasan, M. V. (1985). Shouldn't movement detection necessarily be "colour-blind"? Vision Research, 25, 997-1000.

Troscianko, T. (1987). Perception of random-dot symmetry and apparent movement at and near isoluminance. Vision Research, 27, 547-554.

Troscianko, T. \& Fahle, M. (1988). Why do isoluminant stimuli appear slower? Journal of the Optical Society of America, A5, 871-880. 